\title{
Inventario de mamíferos no voladores en remanentes de bosque seco tropical en el valle del río Cauca, Cartago, Colombia
}

\author{
Julián Ricardo Henao-Isaza* (iD), Juan Esteban Payán-Montoya (iD, Alex Mauricio

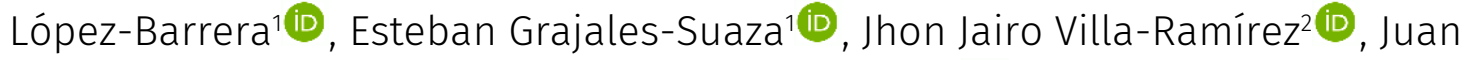 \\ Manuel Betancourt-Torres ${ }^{\text {iD }}$
}

\begin{abstract}
1 Grupo de investigación Biología de la conservación y biotecnología, Corporación Universitaria de Santa Rosa de Cabal, vereda El Jazmín km. 4 vía Santa Rosa de Cabal - Chinchiná, Colombia.

2 Oficina Asesora de Calidad y Medioambiente, Empresas Municipales de Cartago ESP, Calle 13 No $5 a-35$ 2do Piso, Cartago, Colombia.

* Correspondencia: ricardj82@gmail.com
\end{abstract}

\section{Resumen}

El estudio de la diversidad local de los mamíferos en el bosque seco tropical (BST) es importante, debido al valor cultural que tienen para las comunidades locales como fuente de alimento y medicina. Además, la información sobre estos ensamblajes es útil para el manejo del BST, uno de los ecosistemas más amenazados de Colombia cuyos remanentes constituyen importantes albergues para la fauna. El objetivo de este trabajo fue caracterizar los mamíferos medianos no voladores del "Bosque seco tropical Hernán Victoria Mena", municipio de Cartago (Valle del Cauca). El trabajo de campo se desarrolló durante 9 días en dos fases, empleando técnicas para la detección directa e indirecta (fototrampeo, trampas de huellas, recorridos libres y entrevistas). Se acumularon 130 registros correspondientes a 19 especies de mamíferos. Algunas están citadas en la lista roja de la IUCN y el libro rojo: Lontra longicaudis, Aotus lemurinus y Cabassous centralis. Los resultados presentados en este documento serán utilizados como línea base para implementar iniciativas de manejo por parte de los propietarios del predio.

Palabras clave: Inventario, Biodiversidad, Conservación biológica, Mammalia, Riqueza de especies.

\begin{abstract}
The study of the local diversity of mammals in the TDF is important, due to the cultural value they have for local communities as a source of food and medicine. In addition, the information on these assemblies is useful for the management of the tropical dry forest, one of the most threatened ecosystems in Colombia whose remnants constitute important shelters for fauna. The objective of this work was to characterize the non-flying medium mammals of the "Hernán Victoria Mena Tropical Dry Forest", Cartago municipality (Valle del Cauca). The fieldwork was carried out during 9 days in two phases, using techniques for direct and indirect detection (camera trapping, foot print traps, transects and interviews). A total of 130 records corresponding to 19 mammal species were
\end{abstract}


accumulated. Some are cited on the IUCN Red List and Red Book: Lontra longicaudis, Aotus lemurinus, and Cabassous centralis. The results presented in this document will be used as a baseline to implement management initiatives by the owners of the property.

Key words: Inventory, Biodiversity, Biological Conservation, Mammalia, Species richness.

El bosque seco tropical (BST) tiene una marcada estacionalidad de lluvias (IAvH, 2019), con 250-2000 mm de precipitación al año y una estación seca de 3-4 meses (Grainger 1996; Mayaux et al. 2005; Miles et al. 2006). En Colombia se encuentra en seis regiones, entre ellas los valles interandinos del río Magdalena y Cauca (IAvH 1998), donde persiste en pequeños remanentes aislados entre sí (Mendoza-C 1999). Presenta bajas tasas de crecimiento vegetal en comparación con los bosques húmedos, haciéndolos más susceptibles a la perturbación (Murphy \& Lugo 1986).

El BST es uno de los ecosistemas más amenazado de Colombia y considerado en peligro crítico (Etter et al. 2017), donde solo el $0,4 \%$ de la superficie conserva su cobertura (IAvH 2019). A pesar de esta condición, algunos estudios han revelado que estos remanentes constituyen importantes albergues para la fauna, resaltando especies con alto valor biológico y socioeconómico (Orjuela-C \& Jiménez 2004; Vela-Vargas \& Pérez-Torres 2012).

Los estudios relacionados con el BST se centran en zonas muy intervenidas y geográficamente pequeñas, por lo cual no se dispone de inventarios completos que correlacionen la diversidad y ecología de la mastofauna en todas las regiones del BST en Colombia (Pizano \& García 2014). Entender estos procesos es necesario para orientar la toma de decisiones y las estrategias para su conservación.

Los mamíferos terrestres son especies con alto valor cultural en las comunidades locales, debido a su uso con fines alimenticios, mágicos y de medicina tradicional (Parra-Colorado et al. 2014; Racero-Casarrubia \& González-Maya 2014; Tinoco-Sotomayor et al. 2016). En otros casos, se perciben como animales conflictivos o perjudiciales, por matar la fauna doméstica, por ejemplo, el zorro, la comadreja o la zarigüeya y son cazados como represalia (Deustua-Aris et al. 2008).

Así mismo, los mamíferos terrestres tienen un alto valor ecológico. Por una parte, las especies frugivoras dispersan las semillas de las plantas que se alimenta; de esta manera, se involucran en el proceso de sucesión ecológica (Stoner et al. 2007). Las especies herbivoras y las fosoriales generan cambios directos en la vegetación que determinan la dirección de la sucesión ecológica, tal como el ramoneo selectivo de especies vegetales y cambios en la topografia del suelo (Severtsov 2013). Las especies carnívoras, así como las demás, están involucradas en el flujo de energía y el ciclo de materia, aunque su papel es complejo debido al comportamiento omnívoro de los consumidores primarios y secundarios (Severtsov 2013).

El objetivo de este trabajo fue caracterizar los mamíferos medianos no voladores en el predio denominado "Bosque seco tropical Hernán Victoria Mena", perteneciente a las Empresas Municipales de Cartago E.S.P (lat 4,75365, long -75,89435 WGS84). Está localizado en el extremo nororiental del casco urbano del municipio de Cartago (Valle del Cauca), adyacente al cauce del río La Vieja con una altitud de 940 msnm (Figura 1). De acuerdo con el Plan de Ordenamiento Territorial, el predio se encuentra en la categoría de "suelos de protección" y parte de sus bosques constituyen área forestal protectora del río La Vieja (Municipio de Cartago 2019). 


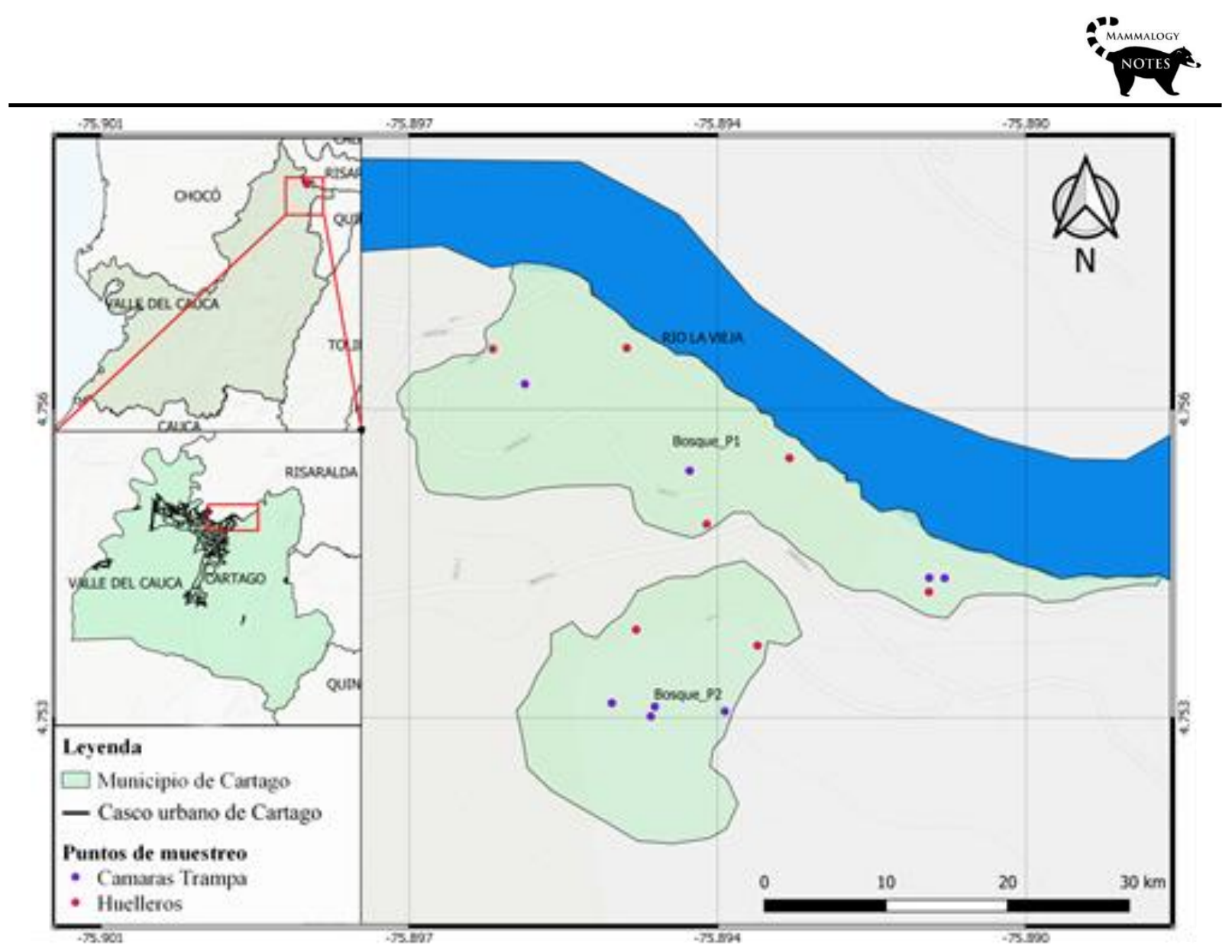

FIGURA 1. Localización del área de estudio "Bosque seco tropical Hernán Victoria Mena" de las Empresas Municipales de Cartago E.S.P. En la figura se muestra el departamento Valle del Cauca y el municipio de Cartago, los fragmentos del bosque seco tropical Hernán Victoria Mena y los puntos de muestreo de las técnicas utilizadas: cámaras trampa (puntos azules) y huelleros (puntos rojos).

El trabajo de campo se desarrolló durante 9 días en dos fases, la primera entre el 26 de noviembre y el 01 de diciembre y la segunda entre el 06 y 08 de diciembre de 2019. Se empleó una combinación de técnicas para la detección directa e indirecta. Se instalaron 7 trampas de huellas de $1 \mathrm{~m}^{2}$ (Figura 2, A), con atrayentes olfativos naturales, tal como sardina, maíz, maní crudo (Aranda 2000). Las huellas fueron registradas mediante fotografias y algunas fueron colectadas utilizando un molde de yeso odontológico (Aranda 2012). Se emplearon 8 estaciones de fototrampeo, cada una con una cámara ubicada en árboles rectos a $30 \mathrm{~cm}$ del suelo (Díaz-Pulido \& Payán 2012). Las cámaras se instalaron dentro de la vegetación boscosa, en presuntos pasos de fauna y cerca de cuerpos de agua (Figura 2, B). El esfuerzo en trampas de huellas fue 24 noches/trampa y en cámaras trampa fue 48 noches/cámara.

Se realizaron recorridos libres para la detección diurna (16:00-18:00) y nocturna (21:000:00) de mamíferos y sus rastros y realizando pausas de 5-10 minutos en sitios estratégicos (árboles fructificados, riberas del río, etc.) (Gómez 2014). Los recorridos se realizaron por caminos, carreteras y áreas semiabiertas (Figura 2, C), inspeccionando los diferentes estratos (suelo, sotobosque, dosel) en los dos fragmentos de bosque del área de estudio (Figura 1). Cada fragmento se recorrió en 3 ocasiones. Los individuos observados fueron 
registrados en libretas de campo con información estándar, como hora, tipo de registi coordenadas, altitud, entre otros.

De manera complementaria, se realizó un recorrido por vía fluvial por un único margen del río La Vieja, con lo cual se abarcó una distancia aproximada de 1,7 Km (Figura 2, D). Este recorrido se realizó de manera oportunista al final del muestreo y tuvo como finalidad complementar los datos obtenidos, principalmente para la búsqueda de rastros de especies acuáticas y semiacuáticas y el reconocimiento de sitios potenciales de refugio.

Por último, se realizaron 10 entrevistas informales al personal que permanece en el área de estudio para complementar los datos primarios (Medina et al. 2012). Estas consisten en mostrar ilustraciones de mamíferos neotropicales para que las personas señalen aquellos que creen haber observado y ofrezcan una descripción del encuentro (Figura 2, E), indicando el hábitat, horario, características externas y el nombre local, este último empleado para elaborar el listado de especies. Para la asignación de los nombres científicos se siguió la nomenclatura taxonómica del Mammal Diversity Database (Burgin et al. 2018).

Se acumularon 130 registros directos e indirectos, que corresponden a 19 especies de mamíferos que habitan en el área de estudio (Tabla 1). Estas especies pertenecen a 14 familias y 6 órdenes taxonómicos, siendo Rodentia (roedores) y Carnivora (carnívoros) los de mayor riqueza (Tabla 1). Los mamíferos Didelphis marsupialis (Figura 2, F) y Dasypus novemcinctus (Figura 2, G) fueron los más incidentes en el muestreo, registrados mediante todas las técnicas en más del $80 \%$ de los días. De la chucha (D. marsupialis) se observaron por lo menos 6 individuos, a nivel del suelo y dosel en el interior del bosque y los bordes; forrajeando mango (Mangifera indica) y mamoncillo (Melicoccus bijugatus). En cuanto al gurre (D. novemcinctus), se observaron por lo menos 5 individuos, solitarios o en pareja, dentro del bosque y en los bordes, cerca de pastizales y carreteras.

De Aotus lemurinus (Figura 2, H) se registraron dos tropas. Una compuesta por tres individuos poco tolerantes a la presencia humana, observados forrajeando Yarumo (Cecropia sp.) y pasiflora (Passiflora sp.). La segunda estaba conformada por cuatro individuos, incluyendo una cría, tolerantes a la presencia humana y forrajeando mango (Mangifera indica). Existen observaciones previas de la presencia de A. lemurinus en bosques intervenidos y con presencia humana (Castaño \& Cardona 2005). Esta tolerancia puede favorecer su supervivencia en hábitats intervenidos, aunque esto no necesariamente garantiza la adaptación evolutiva (Stockwell et al. 2003). Por otro lado, la tolerancia puede aumentar el riesgo de captura, trasmisión de patógenos y ataques por fauna domestica (Castaño \& Cardona 2005; Chapman et al. 2005).

Un total de 9 especies fueron reportadas exclusivamente por entrevistas a la comunidad. La mayoría son probables para el área de estudio, de acuerdo con observaciones personales y reportes previos en municipios cercanos (Castaño et al. 2017; MantillaCastaño et al. 2019) y en el valle geográfico del río Cauca, en su paso por el departamento Valle del Cauca (Rojas-Díaz et al. 2012). Para uno de estos mamíferos (Tamandua mexicana) se obtuvo la evidencia fotográfica del registro (Figura 2, I). 


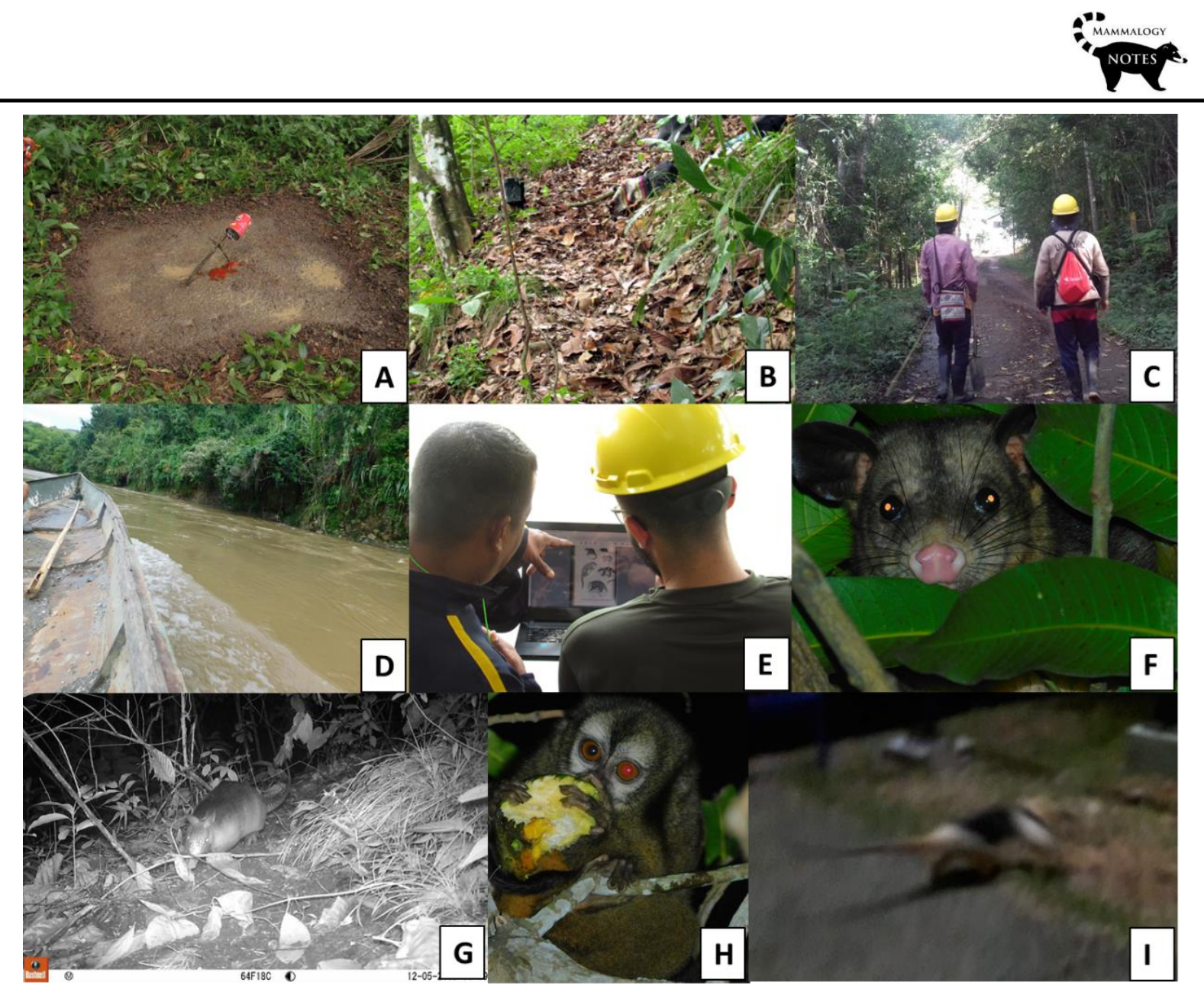

FIGURA 2. Muestreo de mamíferos en el Bosque Seco Tropical Hernán Victoria Mena: trampas huella (A), fototrampeo en presuntos pasos de fauna (B), recorridos terrestres y fluviales (C y D) y entrevistas (E). Registros de algunas especies en el área de estudio: Didelphis marsupialis - Chucha común (F), Dasypus novemcinctus - Gurre (G), Aotus lemurinus - Marteja (H), Tamandua mexicana - Hormiguero, observado por los funcionarios del predio (I).

En cuanto al estado de amenaza global, Lontra longicaudis se encuentra como casi amenazada (NT) y Aotus lemurinus como vulnerable (VU), por otra parte, la especie Cabassous centralis presenta datos deficientes (DD) (Morales-Jiménez \& de la Torre 2008; Tirira et al. 2014; Rheingantz \& Trinca 2015). A nivel nacional, L. longicaudis y A. lemurinus se encuentran en VU y C. centralis en NT (Alberico 2006; Defler \& Rodríguez-Mahecha 2006; Trujillo \& Arcila 2006).

Los remanentes de bosque seco tropical en el área de estudio albergan un ensamblaje compuesto por numerosas especies. Esta información es relevante en la medida que aporta al conocimiento de la supervivencia actual de la mastofauna en este ecosistema.

Estudios en escalas más amplias de paisaje sugieren que las áreas de bosque fragmentado poseen una diversidad de mamíferos no voladores mayor que otro tipo de hábitats, como agroecosistemas y bosques de galería (García-Herrera et al. 2015). Sin embargo, la fauna persiste en hábitats degradados por la fragmentación, la acumulación de contaminantes y la ocurrencia de fauna doméstica. Esto genera la reducción en la cantidad de hábitat disponible para las poblaciones y provoca cambios en las propiedades del ecosistema (Fahrig 2003). 
TABLA 1. Listado de especies de mamíferos no voladores registrados en el Bosque seco tropical Hernán Victoria Mena. Los nombres locales fueron asignados de acuerdo con las entrevistas de la comunidad local. Abreviaciones: CAM (Cámara trampa), ENT (entrevista), OBS (observación directa), RAS (rastro), N/D (no determinado).

\begin{tabular}{|c|c|c|c|}
\hline TAXA & NOMBRE LOCAL & TIPO DE REGISTRO & IUCN \\
\hline \multicolumn{4}{|l|}{$\begin{array}{l}\text { Orden Carnivora } \\
\text { Canidae }\end{array}$} \\
\hline Cerdocyon thous & $\begin{array}{l}\text { Lobito, zorro, zorrillo, perro } \\
\text { de monte }\end{array}$ & CAM, ENT, OBS, RAS & LC \\
\hline \multicolumn{4}{|l|}{ Mustelidae } \\
\hline Eira barbara & $N / D$ & ENT & LC \\
\hline Lontra longicaudis & Nutria & ENT, OBS, RAS & NT \\
\hline Mustela frenata & Comadreja & ENT, OBS & LC \\
\hline \multicolumn{4}{|l|}{ Procyonidae } \\
\hline Potos flavus & Perro de monte, mico & ENT, OBS & LC \\
\hline Procyon cancrivorus & $N / D$ & ENT & LC \\
\hline \multicolumn{4}{|l|}{$\begin{array}{l}\text { Orden Cingulata } \\
\text { Dasvpodidae }\end{array}$} \\
\hline Cabassous centralis & Cola de trapo, gurre & ENT, RAS & DD \\
\hline Dasypus novemcinctus & Armadillo, gurre & CAM, ENT, OBS, RAS & LC \\
\hline \multicolumn{4}{|l|}{$\begin{array}{l}\text { Orden Didelphimorphia } \\
\text { Didelphidae }\end{array}$} \\
\hline Didelphis marsupialis & Chucha, zarigüeya & CAM, ENT, OBS, RAS & LC \\
\hline Indeterminado sp 1 & $\begin{array}{l}\text { Chucha mantequera, } \\
\text { mantequera, marteja }\end{array}$ & ENT, RAS & LC \\
\hline \multicolumn{4}{|l|}{ Orden Lagomorpha } \\
\hline \multicolumn{4}{|l|}{ Leporidae } \\
\hline Sylvilagus brasiliensis & Conejo & ENT & LC \\
\hline \multicolumn{4}{|l|}{ Orden Pilosa } \\
\hline Bradypus variegatus & $s / n$ & ENT & LC \\
\hline \multicolumn{4}{|l|}{ Megalonychidae } \\
\hline Choloepus hoffmanni & Perezoso & ENT & LC \\
\hline \multicolumn{4}{|l|}{ Myrmecophagidae } \\
\hline Tamandua mexicana & Hormiguero & ENT & LC \\
\hline \multicolumn{4}{|l|}{ Orden Primates } \\
\hline Aotus lemurinus & Marteja, mico & ENT, OBS & VU \\
\hline \multicolumn{4}{|l|}{$\begin{array}{l}\text { Orden Rodentia } \\
\text { Caviidae }\end{array}$} \\
\hline Hydrochoerus sp. & Chigüiro & & $N / D$ \\
\hline \multicolumn{4}{|l|}{ Cuniculidae } \\
\hline Cuniculus paca & Guagua & CAM, ENT, OBS, RAS & LC \\
\hline \multicolumn{4}{|l|}{ Dasyproctidae } \\
\hline Dasyprocta punctata & Guatín & CAM, ENT, OBS, RAS & LC \\
\hline \multicolumn{4}{|l|}{ Erethizontidae } \\
\hline $\begin{array}{l}\text { Coendou rufescens } \\
\text { Indeterminado }\end{array}$ & $N / D$ & ENT & LC \\
\hline Indeterminado sp 2 & Ratón & CAM & $N / D$ \\
\hline Indeterminado sp 3 & Ratón & OBS & $N / D$ \\
\hline \multicolumn{4}{|l|}{ Sciuridae } \\
\hline Sciurus granatensis & Ardilla, ardita & ENT, OBS & LC \\
\hline
\end{tabular}


Estos cambios constituyen presiones selectivas para las especies que observamos actualmente, pero desconocemos su capacidad de adaptación en el área de estudio. Esto sucede porque las poblaciones responden ante las perturbaciones con cambios en las características fenotípicas, pero no siempre coinciden con el "óptimo" para tener éxito reproductivo y cuando esto ocurre, disminuyen gradualmente entre generaciones hasta su extinción (Stockwell et al. 2003). Por lo anterior, no se puede garantizar la supervivencia de las especies reportadas en el área de estudio a mediano o largo plazo, a menos que se tomen acciones de manejo y conservación para este ecosistema.

Finalmente, cabe mencionar que los resultados fueron socializados con la administración del predio y se reconoció la importancia de realizar acciones para conservar los mamíferos presentes en el área, tanto por su valor ecológico como por su valor cultural y el carisma que generan a la comunidad en general. Esto es relevante, en la medida que el "Bosque Seco Tropical Hernán Victoria Mena" constituye un hábitat para las poblaciones de estas especies en un ambiente altamente intervenido.

\section{AGRADECIMIENTOS}

Los autores expresan su gratitud hacia a las Empresas Municipales de Cartago E.S.P., que propuso y financió el estudio de fauna en su predio. Así mismo, gratitud hacia las personas de la comunidad, quienes participaron en las entrevistas de manera voluntaria y emotiva. Finalmente, a los evaluadores y editores de la revista quienes aportaron sus valiosos conocimientos para mejorar este manuscrito.

\section{REFERENCIAS}

Alberico M. 2006. Armadillo coletrapo centroamericano, Cabassous centrales. In: Rodríguez-M JV, Alberico M, Trujillo F, Jorgenson J, editores. Libro Rojo de Los Mamíferos de Colombia. Bogotá DC: Conservación Internacional Colombia, Ministerio del Medio Ambiente, Vivienda y Desarrollo Territorial. p. 324-327.

Aranda JM. 2012. Manual para el rastreo de mamíferos de México, Primera edición. México DF: Comisión Nacional para el Conocimiento y Uso de la Biodiversidad (CONABIO).

Aranda JM. 2000. Huellas y otros rastros de los mamíferos grandes y medianos de México, Primera ed. Veracruz, México: Instituto de Ecología A.C.

Burgin CJ, Colella JP, Kahn PL, Upham NS. 2018. How many species of mammals are there? Journal of Mammalogy. 99:1-14. https:// doi.org/10.1093/jmammal/gyx147

Castaño JH, Cardona DM. 2005. Presencia Del Mono Nocturno Andino (Aotus lemurinus I. GeoffroySt. Hilaire, 1843) En Fragmentos De Bosque De La Cuenca Media Del Río Cauca. Boletín Científico Museo de Historia Natural Universidad de Caldas. 9:111-117.

Castaño JH, Torres DA, Rojas-Díaz V, Saavedra-Rodríguez CA, Pérez-Torres J. 2017. Mamíferos del departamento de Risaralda, Colombia. Biota Colombiana. 18:239-254. https:// doi.org/10.21068/c2017.v18n02a16

Chapman CA, Gillespie TR, Goldberg TL. 2005. Primates and the ecology or their infectious diseases: how will anthropogenic change affect host-parasite interactions? Evolutionary Anthropology. 14:134-144.

Defler TR, Rodríguez-Mahecha JV. 2006. Mico de noche andino, complejo Aotus lemurinus, in: Rodríguez-M JV, Alberico M, Trujillo F, Jorgenson J editores, Libro Rojo de Los Mamíferos de 
Colombia. Bogotá DC: Conservación Internacional Colombia, Ministerio del Medio Ambiente, Vivienda y Desarrollo Territorial. p. 212-216.

Deustua-Aris I, Williams-León De Castro M, Vásques-Ruesta P. 2008. Relaciones entre los pobladores rurales y los carnivoros altoandinos del distrito de Anco, Centro, sur del Perú. Ecología Aplicada. 7:43-48.

Díaz-Pulido A, Payán E. 2012. Mamíferos grandes, metodología de evaluación de censos por transectos. In: Manual de Fototrampeo: Una Herramienta de Investigación Para La Conservación de La Biodiversidad En Colombia. Bogotá DC: Instituto de Investigaciones de Recursos Biológicos Alexander von Humboldt. Panthera Colombia. p. 32.

Etter A, Andrade A, Saavedra K, Amaya P, Arevalo P, Cortés J, Pacheco C, Soler D. 2017. Lista Roja de Ecosistemas de Colombia (Vers.2.0). Bogotá DC: Pontificia Universidad Javeriana, Conservación Internacional https:// doi.org/10.13140/RG.2.2.10861.08165

Fahrig L. 2003. Effects of Habitat Fragmentation on Biodiversity. Annual Review of Ecology, Evolution, and Systematics. 34:487-515. https:/ / doi.org/10.1146/annurev.ecolsys.34.011802.132419

García-Herrera LV, Ramírez-Fráncel LA, Reinoso Flórez G. 2015. Mamíferos en relictos de bosque seco tropical del Tolima, Colombia. Mastozoología Neotropical. 22:11-21.

Gómez F. 2014. Metodologías para el monitoreo de la biodiversidad en la Amazonía. In: Programa de Monitoreo de la Biodiversidad, editores. Metodologías Para el Monitoreo de la Biodiversidad en la Amazonía. Perú: Consorcio Camisea. p. 111-122. https://doi.org/10.1088/1748$9326 / 9 / 3 / 034012$

Grainger A. 1996. An evaluation of the FAO tropical forest resource assessment, 1990. Geogr. J. 162:7379. https://doi.org/10.2307/3060217

IAvH. 2019. Bosques secos tropicales en Colombia [WWW Document]. URL http:/ / www.humboldt.org.co/en/research/projects/developing-projects/item/158bosques-secos-tropicales-en-colombia

IAvH. 1998. Informe nacional sobre el estado de la biodiversidad 1997. Bogotá DC: Ministerio del Medio Ambiente; PNUMA; Programa de la Naciones Unidas para el Medio Ambiente.

Mantilla-Castaño JC, Manzano-Valencia A, Henao-Isaza JR, Torres-Arboleda DA, Pareja-Márquez IM, González-Montenegro GE, Carranza-Quiceno JA, Castaño JH, Cortés S. 2019. Pelos, plumas y escamas en las cuencas bajas de los ríos Cestillal y Barbas. Pereira, Colombia: Asociación Ambiental Chinampa.

Mayaux P, Holmgren P, Achard F, Eva H, Stibig HJ, Branthomme A. 2005. Tropical forest cover change in the 1990s and options for future monitoring. Philosophical Transactions of the Royal Society B: Biological Sciencies 360:373-384. https:// doi.org/10.1098/rstb.2004.1590

Medina CE, Zeballos H, López E. 2012. Diversidad de mamíferos en los bosques montanos del Valle de Kcosñipata, Cusco, Perú. Mastozoología Neotropical 19:85-104.

Mendoza-C H. 1999. Estructura y riqueza florística del bosque seco tropical en la región Caribe y el Valle del río Magdalena, Colombia. Caldasia 21:70-94.

Miles L, Newton AC, DeFries RS, Ravilious C, May I, Blyth S, Kapos V, Gordon JE. 2006. A global overview of the conservation status of tropical dry forests. Journal of Biogeography 33:491-505. https:// doi.org/10.1111/j.1365-2699.2005.01424.x

Morales-Jiménez AL, de la Torre S. 2008. Aotus lemurinus. The IUCN Red List of Threatened Species 2008 e.T1808A7651803. URL https://dx.doi.org/10.2305/IUCN.UK.2008.RLTS.T1808A7651803.en Accessed on 28 May 2020 
Municipio de Cartago. 2019. POT VIGENTE. URL https: / www.cartago.gov.co/pot-vigente Accessed on 21 May 2020

Murphy PG, Lugo AE. 1986. Ecology of tropical dry forest. Annual Review of Ecology and Systematics 17:67-88. https:// doi.org/10.1146/annurev.es.17.110186.000435

Orjuela OJ, Jiménez G. 2004. Mamíferos en diferentes tipos de coberturas y carretera, finca Hacienda Cristales, área Cerritos - La Virginia, Municipio De Pereira, Departamento de Risaralda, Colombia. Universitas Scientiarum 9:87-96.

Parra-Colorado JW, Botero-Botero Á, Saavedra-Rodríguez CA. 2014. Percepción y uso de mamíferos silvestres por comunidades campesinas andinas de Génova, Quindío, Colombia. Boletín Científico del Centro de Museos 18:78-93.

Pizano C, García H. 2014. El Bosque Seco Tropical en Colombia. Bogotá DC: Instituto de Investigación de Recursos Biológicos Alexander von Humboldt (IAvH).

Racero-Casarrubia J, González-Maya JF. 2014. Inventario preliminar y uso de mamíferos silvestres por comunidades campesinas del sector oriental del cerro Murrucucú, municipio de Tierralta, Córdoba, Colombia. Mammalogy Notes 1:25-28.

Rheingantz ML, Trinca CS. 2015. Lontra longicaudis. The IUCN Red List of Threatened Species 2015: e.T12304A21937379. https://dx.doi.org/10.2305/IUCN.UK.2015-2.RLTS.T12304A21937379.en. Accessed on 20 May 2020.

Rojas-Díaz V, Reyes-Gutiérrez M, Alberico MS. 2012. Mamíferos (Synapsida, Theria) del Valle del Cauca, Colombia. Biota Colombiana. 13:99-117. https:/ / doi.org/10.21068/ bc.v13i1.259

Severtsov AS. 2013. The significance of vertebrates in the structure and functioning of ecosystems. Biology Bulletin 40:571-579. https:// doi.org/10.1134/S1062359013070054

Stockwell CA, Hendry AP, Kinnison MT. 2003. Contemporary evolution meets conservation biology. Trends in Ecology and Evolution 18:94-101. https:/ / doi.org/10.1016/S0169-5347(02)00044-7

Stoner KE, Riba-Hernández P, Vulinec K, Lambert JE. 2007. The role of mammals in creating and modifying seedshadows in tropical forests and some possible consequences of their elimination. Biotropica 39:316-327. https:// doi.org/10.1111/j.1744-7429.2007.00292.x

Tirira DG, Díaz-N J, Superina M, Abba AM. 2014. Cabassous centralis. The IUCN Red List of Threatened Species 2014: e.T3412A47437304. https://dx.doi.org/10.2305/IUCN.UK.20141.RLTS.T3412A47437304.en Accessed on 28 May 2020.

Trujillo F, Arcila D. 2006. Nutria neotropical, Lontra longicaudis. In: Rodríguez-M JV, Alberico M, Trujillo F, Jorgenson J, editores. Libro Rojo de Los Mamíferos de Colombia. Bogotá DC: Conservación Internacional Colombia, Ministerio del Medio Ambiente, Vivienda y Desarrollo Territorial. p. 251-255.

Vela-Vargas IM, Pérez-Torres J. 2012. Murciélagos asociados a remanentes de bosque seco tropical en un sistema de ganadería extensiva (Colombia). Chiroptera Neotropical 18:1089-1100.

Editor: José F. González-Maya

Recibido 2020-04-16

Revisado: 2020-06-10

Aceptado 2020-07-24

Publicado 2020-09-28 